\title{
Calidad de vida en adolescentes de 14 a 18 años con fibrosis quística en la fundación de fibrosis quística, Paraguay
}

\section{Quality of life in adolescents aged 14 to 18 years with cystic fibrosis at the cystic fibrosis foundation, Paraguay}

\author{
Romina Paola Radice ${ }^{1}$, Oscar Augusto Radice ${ }^{2}$, Mélani Rebeca Radice $^{3}$, César Augusto \\ Radice $^{4}$
}

\section{RESUMEN}

Introducción: La fibrosis quística es una enfermedad crónica de relativa frecuencia, con repercusión en la calidad de vida de quienes la padecen. El objetivo de este estudio es analizar la calidad de vida de los adolescentes de 14 a 18 años con fibrosis quística, en la Fundación de Fibrosis Quística del Paraguay. Materiales y Métodos: El estudio se realizó en adolescentes 14 a 18 años, ambulatorios de la Fundación de Fibrosis Quística del Paraguay, que completaron en forma autoadministrada el cuestionario CFQ-R. Se aplicó un diseño observacional, descriptivo, prospectivo y de corte transversal. La carga, análisis y edición de los datos se realizó mediante el Scoring Program del CFQ-R. Resultado: La media de edad fue de 15 años y la desviación estándar de 1,52, con un $71,4 \%$ de mujeres y un $28,6 \%$ varones. El nivel de educación que predominó fue la media. El resultado respecto a los dominios según los aspectos generales, la puntuación media más alta correspondió a estado emocional, y la media con menor puntuación fue vitalidad, respecto a los dominios en los aspectos específicos, la puntuación media más alta correspondió a síntomas digestivos, y la media con menor puntuación fue carga del tratamiento. Conclusión: La menor puntuación correspondió a la vitalidad en el aspecto general y la de menor puntuación en los específicos fue la carga del tratamiento.

Palabras claves: Calidad de vida, fibrosis quística, adolescentes.

\begin{abstract}
Introduction: Cystic fibrosis is a chronic disease of relative frequency, with repercussions on the quality of life of those who suffer from it. The objective of this study was to analyze the quality of life of adolescents aged 14 to 18 years with cystic fibrosis, at the Cystic Fibrosis Foundation of Paraguay. Materials and Methods: The study was carried out in adolescents 14 to 18 years old, who were outpatients at the Cystic Fibrosis Foundation of Paraguay, and who completed the CFQ-R questionnaire on their own. An observational, descriptive, prospective and crosssectional design was applied. Data loading, analysis and editing was performed using the CFQ-R Scoring Program. Results: The mean age was 15 years and the standard deviation was 1.52 , with $71.4 \%$ women and $28.6 \%$ men. The level of education that predominated was high school. Regarding general domain scores, the highest mean score corresponded to emotional state, and the mean with the lowest score was vitality. With regards to specific domains, the highest mean score corresponded to digestive symptoms, and the lowest mean score was treatment burden. Conclusion: The lowest score corresponded to vitality in the general domain and the lowest score in the specific domain was the treatment burden.
\end{abstract}

Keywords: Quality of life, cystic fibrosis, adolescents.

\footnotetext{
Consultora independiente. Ciudad del Este, Paraguay.

${ }^{2}$ Universidad Nacional del Este, Facultad de Ciencias de la Salud. Ciudad del Este, Paraguay.

${ }^{3}$ Fundación Tesãi. Ciudad del Este, Paraguay.

${ }^{4}$ Universidad Nacional del Este, Facultad de Ciencias de la Salud, Centro de Investigaciones Médicas. Ciudad del Este, Paraguay.

Correspondencia: Cesar Radice Correo: cesarradice@hotmail.com

Conflicto de interés: Los autores declaran no poseer conflicto de interés.

Recibido: 02/09/2020 Aceptado: 25/09/2020

Doi: https://doi.org/10.31698/ped.47032020007
} 


\section{INTRODUCCIÓN}

La fibrosis quística es una enfermedad genética autosómica recesiva de las glándulas exocrinas. Está causada por mutaciones en el gen que codifica el regulador de la conductancia transmembrana en la fibrosis quística, que se manifiesta en varios órganos como pulmones, páncreas, tracto biliar, y las glándulas sudoríparas. La misma se caracteriza por disfunción secretora epitelial, la cual da lugar a obstrucción de las vías aéreas, infecciones crónicas del tracto respiratorio, insuficiencia pancreática, mala digestión, depleción salina y agotamiento producidas por el calor ${ }^{(1-3)}$.

La fibrosis quística (FQ) es uno de los trastornos más frecuentes que amenaza la vida, en el mundo occidental. Es una patología con alta prevalencia entre los caucásicos, con incidencia en el nacimiento que van desde 1 en 2500 (recién nacidos en Finlandia) a 1 en 1800 (recién nacidos en Eslovaquia)(4), la mayoría de estos pacientes desarrollan enfermedad pulmonar crónica e insuficiencia pancreática ${ }^{(5)}$.

En América latina se estima que la incidencia en recién nacidos (RN) en Brasil varía entre 1 de cada 1.600 a 14.000, los demás países oscilan entre 1 de cada 1.600 a 8.000 , se cree que estos no representan a los números reales, debido al diagnóstico insuficiente, que sigue siendo un desafío para nuestros países ${ }^{(6)}$.

Los estudios genéticos hechos en Paraguay arrojaron el siguiente resultado en cuanto a la composición promedio del paraguayo: 55,0 \% europea; $38,0 \%$ amerindia; $8,0 \%$ africana ${ }^{(7)}$, estos datos nos hace pensar que tendremos el mismo rango que los otros países de la región.

La proteína reguladora de la conductancia transmembrana, esta proteína actúa como un canal de cloro controlado por AMPc en forma directa al cloro, de manera indirecta al agua y sodio, lo cual hace que se forme un moco anormal y espeso principalmente en pulmones, páncreas, intestino, hígado, glándulas sudoríparas, y conductos deferentes en los varones impidiendo su función ${ }^{(8)}$. Este moco espeso facilita la colonización bacteriana y liberación de mediadores proinflamatorios ${ }^{(9)}$. Esto produce falta de apetito, mala absorción intestinal y falla de medro(10).

La función pulmonar es la que establece el estándar de salud en los pacientes con $\mathrm{FQ}^{(11)}$, en consecuencia, la expansión de la misma suele ser la que más repercute en la calidad de vida de estas personas limitando sus actividades en intensidad y tiempo, la mala tolerancia a ambientes poco saludables que los obliga al aislamiento, que genera ansiedad y depresión dominios que se asocian a una calidad de vida deteriorada, este último dominio tiene repercusión en el ausentismo escolar, laboral y productividad $^{(12)}$.

Se ha producido en los últimos 20 años mejoras en la salud de estas personas en forma permanente, continua y sorprendente en lo referente a calidad de vida y supervivencia, esto se debió a la disponibilidad y accesibilidad de centros multidisciplinarios con especialistas en $\mathrm{FQ}$, a la realización de diagnóstico precoz, y lógicamente a los avances del tratamiento y sostenibilidad de los mismos mediante instituciones de ONGs, además de las públicas ${ }^{(13)}$.

Debido a la relativa frecuencia de la patología, es importante contar con conocimientos de algunos indicadores de calidad de vida de personas con fibrosis quística, con el fin de lograr mejorar el estado de salud y calidad de vida.

El objetivo de este estudio es analizar la calidad de vida de los adolescentes de 14 a 18 años con fibrosis quística, en la Fundación de Fibrosis Quística del Paraguay.

\section{MATERIALES Y MÉTODOS}

\section{Diseño del estudio y población}

El estudio se realizó en adolescentes de 14 a 18 años, ambulatorios de la Fundación de Fibrosis Quística del Paraguay, durante el periodo de setiembre a octubre de 2017. 
El trabajo se realizó bajo un enfoque cuantitativo. Se aplicó un diseño observacional, descriptivo, prospectivo y de corte transversal. Se incluyeron pacientes de 14 a 18 años de edad que se encuentran en el programa de la Fundación de Fibrosis Quística del Paraguay, todos con diagnóstico confirmado y que comprendieran adecuadamente el cuestionario. La población estuvo constituida por 7 adolescentes de 14 a 18 años cuyos padres y participantes decidieron participar. El muestreo fue no probabilístico de tipo intencional.

\section{Instrumento}

El reclutamiento se realizó a través de reuniones personalizadas con los pacientes de 14 a 18 años y sus padres, el investigador y los miembros del Comité de la Fundación de Fibrosis Quística del Paraguay (CFFQP), quienes tienen la confianza de sus pacientes. Estos seguían sus controles periódicos en la institución referida.

La recolección de datos se realizó mediante la aplicación del instrumento que fue la versión del Cuestionario de Fibrosis Quística-Revised-Teen/ Adult (CFQ-R 14+), es un instrumento de CVRS real y bien validado para individuos con FQ de 14 años o más. El CFQ fue desarrollado inicialmente en Francia, posteriormente traducida y validada en su versión inglesa, ésta sufrió varias modicaciones convirtiéndose en su versión revisada, que ha sido traducida al castellano y validada transculturalmente para uso en la población hispanohablante de Estados Unidos por Quittner et al. ${ }^{(14)}$. El grupo de Oliveira et al. ${ }^{(15)}$ ha adaptado la escritura de algunos itemes sin cambiar el concepto de las preguntas, la versión final fue valorada por otros miembros de la Unidad de FQ del Hospital Regional Universitario Carlos Haya, Málaga, España, en relación a su comprensión y de 8 pacientes voluntarios que determinaron la idoneidad de la redacción.

El CFQ-R 14+ evalúa la información demográfica y los doce dominios de funcionamiento (6 generales y 6 especificos) Los seis generales son: capacidad física, limitaciones de rol, vitalidad, percepción de la salud, estado emocional, aislamiento social. Los seis específicos son: imagen corporal, problemas con alimentación, carga del tratamiento, problemas de peso, síntomas respiratorios y síntomas digestivos.
Cada pregunta es una variable única y las respuestas se hacen usando escala de calificación Likert cuyos valores de puntajes van de 1 a 4 que incluyen frecuencia (siempre, a menudo, a veces, nunca), intensidad (mucho, algo, un poco, nada), y escalas verdadero-falso (muy cierto, algo verdadero, algo falso, muy falso). Estos valores se suman para generar una puntuación de dominio y se estandariza. Las puntuaciones estandarizadas se traducen en valores entre 0 y 100. Los mayores puntajes corresponden al mejor resultado. Las puntuaciones de cada dominio se calculan si se completan al menos $2 / 3$ de las preguntas. No existe una puntuación total del cuestionario que integre todos los dominios ${ }^{(15)}$.

\section{Variables en estudio}

Las variables en estudio son: edad, sexo, nivel de educación (media o universitaria) y calidad de vida con sus dominios respectivos.

\section{Método estadístico}

La carga, análisis y edición de los datos se realizó mediante el Scoring Program $®$ del Cuestionario de Fibrosis Quística revisado ${ }^{(15)}$.

El análisis de datos, incluyendo los puntajes de cada dominio se realizó aplicando medidas descriptivas (medias y desvio estándar).

\section{Consideraciones éticas}

El estudio fue aprobado por el Comité de Ética de la Facultad de Ciencias de la Salud. Siguió los estándares de buena práctica clínica de la Declaración de Helsinki y se desarrolló de acuerdo con las regulaciones paraguayas vigentes: Resolución No. 146 del 8 de marzo de 2012, Ministerio de Salud Pública y Bienestar Social $(\mathrm{MSPyBS})^{(16)}$ que clasifica este tipo de estudio en la categoría de investigación libre de riesgo.

\section{RESULTADOS}

Durante el periodo de estudio se registraron 7 pacientes de 14 a 18 años de edad, que se encuentran en el programa de la Fundación de Fibrosis Quística del Paraguay.

Los datos demográficos así como el nivel de educación se muestran en la tabla 1 
Los resultados de cada dominio, expresados en medias con desviación estándar se muestran en el Tabla 2 y 3.

Tabla 1. Característica demográfica, en pacientes con fibrosis quística.

\begin{tabular}{lc}
\hline Parámetros Generales & $(\boldsymbol{n}=\mathbf{7})$ \\
\hline Edad años $(\mathrm{M} \pm \mathrm{DE})$ & $15,00 \pm 1,52$ \\
Hombres n $(\%)$ & $2(28,6)$ \\
Mujeres n $(\%)$ & $5(71,4)$ \\
Educación Media n $(\%)$ & $6(85,7)$ \\
Universitaria n (\%) & $1(14,3)$ \\
\hline
\end{tabular}

Tabla 2. Distribución de los dominios según aspectos generales de calidad de vida en pacientes con fibrosis quística.

\begin{tabular}{clc}
\hline & Dominios & $(\boldsymbol{M} \pm \mathbf{D E})$ \\
\hline & Capacidad Física & $55,10 \pm 21,76$ \\
Calidad de vida de & Limitaciones de Rol & $63,11 \pm 19,74$ \\
adolescentes de 14 & Vitalidad & $54,16 \pm 22,18$ \\
o más años con & Percepción de la Salud & $61,91 \pm 27,87$ \\
Fibrosis Quística & Estado Emocional & $64,28 \pm 20,42$ \\
& Aislamiento Social & $58,48 \pm 22,69$ \\
\hline
\end{tabular}

Tabla 3. Distribución de los dominios según aspectos específicos de calidad de vida en pacientes con fibrosis quística.

\begin{tabular}{clc}
\hline & Dominios & $(\boldsymbol{M} \pm \boldsymbol{D E})$ \\
\hline & Imagen Corporal & $53,71 \pm 24,77$ \\
Calidad de vida de & Problemas con la & $73,01 \pm 27,87$ \\
adolescentes de 14 & Alimentación & \\
o más años con & Carga del Tratamiento & $38,08 \pm 22,11$ \\
Fibrosis Quística & Problemas de Peso & $61,90 \pm 30,00$ \\
& Síntomas Respiratorios & $53,31 \pm 23,02$ \\
& Síntomas Digestivos & $87,31 \pm 9,98$ \\
\hline
\end{tabular}

\section{DISCUSIÓN}

Las puntuaciones extremas se dieron dentro del grupo de los dominios específicos, los síntomas digestivos alcanzaron el mejor resultado y carga del tratamiento fue el que alcanzó resultado más bajo.

Respecto a características demográficas según sexo, hubo mayor frecuencia de mujeres, coincidente con el estudio de Knudsen et al. ${ }^{(17)}$.
El resultado en cuanto a edad es parecido al de Quittner et al. ${ }^{(18)}$. Esta etapa de transición dentro del ciclo vital del ser humano es de difícil manejo porque el paciente tiene un pensamiento crítico e independiente, en consecuencia se da cuenta que padece una enfermedad crónica que lleva a una falla progresiva de órganos sobre todo pulmones y páncreas ${ }^{(18)}$. A pesar de los avances en el diagnóstico y tratamiento es una de las enfermedades crónicas más difícil de manejar, lo que produce mala calidad en el estado de ánimo, afecta el funcionamiento social y ocupacional. Los adolescentes han descripto su calidad de vida, como un complejo equilibrio entre una vida normal y saludable, y sentirse diferente de sus pares sanos ${ }^{(19)}$.

En este estudio la mayoría de los participantes estaban en el nivel medio de educación. El resultado, teniendo en cuenta la proporción, es parecido a lo que ocurrió en España en el año 2011 donde la mayoría estaban cursando el nivel medio, esto se debe a los esfuerzos de los agentes implicados (familiahospital-asociaciones) en concienciar sobre la importancia de que estos adolescentes vivan como sus pares, realizando las mismas actividades y rutinas, de esa manera crecer con sentimientos de mayor seguridad, confianza y autoestima ${ }^{(20)}$, teniendo presente que el mejor nivel educativo proporciona mayor posibilidad de conseguir empleo ${ }^{(5)}$.

Entre los aspectos generales de FQ el dominio de capacidad física fue el de puntaje más bajo, resultado parecido al de Cherobin et al. ${ }^{(21)}$. La actividad física es un dominio prioritario en la calidad de vida de los que padecen $\mathrm{FQ}$, porque previene y retrasa el deterioro de la función pulmonar, además disminuye el aislamiento. Esta actividad debe ser agradable y divertida para que exista adherencia, por lo que es importante trabajar en el mejoramiento del entorno ambiental ${ }^{(22)}$.

El dominio de estado emocional en este estudio tuvo el puntaje más alto, sin embargo otros estudios arrojaron puntajes bajos, estos generalmente acompañan a la capacidad física ${ }^{(5,2)}$. Se encontró una alta prevalencia de angustia psicológica incluyendo a los cuidadores, estos estudios en general son de muestras pequeñas, lo que dificulta la interpretación, pero en general la angustia 
psicológica va acompañada de poca adherencia al tratamiento, peor función pulmonar, frecuentes hospitalizaciones que disminuyen la calidad de vida $^{(23)}$.

Esta diferencia se explica por la edad de los participantes en el estudio, quienes buscaron demostrar independencia y capacidad para tomar sus decisiones, impulsada por una actitud positiva para mantenerse motivados ${ }^{(4)}$.

Las bajas puntuaciones en el dominio de carga de tratamiento en este estudio, revela a este dominio como una barrera para la calidad de vida de los pacientes. En general, los estudios coinciden con este resultado, la cual aumenta con la edad, con factores psicosociales, religiosos y/o espirituales ${ }^{(24)}$.

Los adolescentes van entrando en la etapa adulta, edad en que toman mayor conciencia que se trata de una enfermedad crónica, esto produce la aparición de barreras en el tratamiento, como consecuencia de la complejidad del régimen, olvido, falta de rutina. En este momento es importante el apoyo de organizaciones facilitadoras como clínicas de

\section{REFERENCIAS}

1. MeSH. Cystic Fibrosis [Internet]. NCBI. 1967[Citado 03 Ago 2020]. Disponible en: https://www.ncbi.nlm.nih.gov $/ \mathrm{mesh} /$ ?term=Cystic+Fibrosis

2. Cebrian Pinar M. Fibrosis quística comparativa de tres cuestionarios de calidad de vida [Internet]. Universidad de Valencia; 2015[Citado 08 Ago. 2020]. Disponible en: http://ow.ly/TggU30bI2pJ

3. Rubin BK. Cystic fibrosis 2017-The year in review. Respir Care. 2018;63(2):238-421. doi: https://doi.org/10. 4187/respcare.06052

4. Jamieson N, Fitzgerald D, Singh-Grewal D, Hanson CS, Craig JC, Tong A. Children's experiences of cystic fibrosis: a systematic review of qualitative studies. Pediatrics. 2014;133(6):e1683-97. doi: https://doi.org/10.1542/peds. 2014-0009

5. Martin B, Schechter MS, Jaffe A, Cooper P, Bell SC, Ranganathan S. Comparison of the US and Australian fibrosis quística, siendo lo más importante la autogestión realizada por las mismas ${ }^{(2)}$, acción que es realizada en nuestro país por la Fundación de Fibrosis Quística.

\section{Limitaciones}

Este estudio tiene como limitación la cantidad escasa de participantes; pero fueron las accesibles para obtener datos fidedignos. Sin embargo, puede ser el punto de partida para realizar estudios con grupos mayores.

\section{CONCLUSIONES}

Los dominios según el puntaje en orden decreciente fueron, síntomas digestivos, problemas con la alimentación, estado emocional, limitación de rol, percepción de la salud, problema con el peso, vitalidad, imagen corporal, aislamiento social, síntomas respiratorios, capacidad física y carga de tratamiento. La medición de la calidad de vida permite valorar la enfermedad desde la perspectiva del paciente, aportando información valiosa para la clínica y la investigación.

cystic fibrosis registries: The impact of newborn screening. Pediatrics. 2012;129(2):346-55. doi: http://dx.doi.org/10.15 42/peds.2011-0567

6. Silva Filho LVRF, Castaños C, Ruíz HH. Cystic fibrosis in Latin America-Improving the awareness. J Cyst Fibros. 2016;15(6):791-3. doi: http://dx.doi.org/10.1016/j.jcf. 2016.05.007

7. Ascurra M, Valenzuela A, Salinas M, Rodríguez S, Porzio G, Ortiz L, et al. Incidencia de la fibrosis quística en Paraguay. Pediatr. (Asunción). 2019;46(1):6-10. doi: https://doi.org/10.31698/ped.46012019002

8. Escobar Castro H, Sojo Aguirre A, Gil Ortega D, Nadal Ortega JM. Fibrosis quística. Protoc diagnósticoterapéuticos Gastroenterol Hepatol y Nutr Pediátrica SEGHNP-AEP [Internet]. 2013 [citado 14 May. 2017];2(6):77-84. Disponible en: https://www.aeped.es/ sites/default/files/documentos/fquistica.pdf 
9. Solís Durán MJ. Enfermedad fibroquística. Rev Médica Costa Rica y Centroamérica [Internet]. 2010;67(593):281286. Disponible en: http://www.binasss.sa.cr/revi stas/rmcc/593/art13.pdf

10. Castaños C, Pereyro S, Renteria F, Fernando V, Nadeo J, Paba $\mathrm{P}$, et al. Guía de diagnóstico y tratamiento de pacientes con fibrosis quística: actualización. Arch Argent Pediatría [Internet]. 2014[Citado 15 Ago. 2020];112(3):291192. Disponible en: https://bit.ly/3f00669

11. Sawicki GS, Rasouliyan L, McMullen AH, Wagener JS, McColley SA, Pasta DJ, et al. Longitudinal assessment of health-related quality of life in an observational cohort of patients with cystic fibrosis. Pediatr Pulmonol [Internet]. 2011;46(1):36-44.doi:http://dx.doi.org/10.1002/ppul.21325

12. Pozuelo NM. Influencia de la fisioterapia respiratoria en la calidad de vida de adolescentes y adultos con fibrosis quística. Cantabria: Escuela Universitaria Gimbernat; 2014.

13. Gale Díaz SC. Caracterización de pacientes con Fibrosis Quística diagnosticados por medio de Cloruros en Sudor [Tesis especialización]. [Honduras]: Universidad Nacional Autónoma de Honduras del Valle de Sula; 2015. Disponible en: http://www.bvs.hn/TMVS/pdf/TMVS8 /pdf/TMVS8.pdf

14. Quittner AL, Buu A, Messer MA, Modi AC, Watrous M. Development and validation of the cystic fibrosis questionnaire in the United States: A health-related qualityof-life measure for cystic fibrosis. Chest. 2005;128(4):234754. doi: http://dx.doi.org/10.1378/chest.128.4.2347

15. Olveira G, Olveira C, Gaspar I, Cruz I, Dorado A, PérezRuiz E, et al. Validación de la versión española del cuestionario revisado de calidad de vida para fibrosis quística en adolescentes y adultos (CFQR 14+Spain). Arch Bronconeumol. 2010;46(4):165-75. doi: http://dx.doi.org /10.1016/j.arbres.2010.01.006

16. Ministerio de Salud Pública y Bienestar Social. Resolución S.G. N ${ }^{\circ}$ 146/2012 [Internet]. 2012 [Citado 15 Ago 2020]. Disponible en: https://clacaidigital.info/bitstre am/handle/123456789/780/RES.SG.No\%20146\%20del\%202 $012 \% 20 \mathrm{CON} \% 20 \mathrm{ANEXO}$.pdf? sequence $=5 \&$ isAllowed $=$

17. Knudsen KB, Pressler T, Mortensen LH, Jarden M, Skov M, Quittner AL, et al. Associations between adherence, depressive symptoms and health - related quality of life in young adults with cystic fibrosis. Springerplus. 2016;5(1):1216. doi: http://dx.doi.org/10.11 86/s40064-016-2862-5
18. Quittner AL, Abbott J, Georgiopoulos AM, Goldbeck L, Smith B, Hempstead SE, et al. International Committee on Mental Health in Cystic Fibrosis: Cystic Fibrosis Foundation and European Cystic Fibrosis Society consensus statements for screening and treating depression and anxiety. Thorax. 2016;71(1):26-34. doi: http://dx.doi.org/10.1136/thoraxjnl-2015-207488

19. Jessup M, Parkinson C. "All at sea": the experience of living with cystic fibrosis. Qual Health Res. 2010;20(3):35264. doi: http://dx.doi.org/10.1177/1049732309354277

20. Rios Cortes A, Escribano Montaner A, Soler Juver A, Monfort Gil B, Esteban Romani L, Garriaga García M, et al. Investigación de las personas con fibrosis quística en España 2011: descripción actual y análisis comparativo 1999-2011 [Internet]. Valencia: Federación Española de Fibrosis Quística; 2011[Citado 30 Ago. 2020]. p. 110. Disponible en: https://bit.ly/3fB9Kvh

21. Cherobin I, Ziegler B, Dalcin P. Evaluation of functional capacity and level of physical activity in adolescent and adult patients with cystic fibrosis. Rev Bras Ativ Fís Saúd. 2016;21(2):172-180. doi: https://doi.org/10.12820/rbafs.v.21n2p172-180

22. Denford S, Van Beurden S, O'Halloran P, Williams CA. Barriers and facilitators to physical activity among children, adolescents, and young adults with cystic fibrosis: A systematic review and thematic synthesis of qualitative research. BMJ Open. 2020;10(2):e035261. doi: http://dx.doi.org/10.1136/bmjopen-2019-035261

23. Quittner AL, Goldbeck L, Abbott J, Duff A, Lambrecht $\mathrm{P}$, Solé A, et al. Prevalence of depression and anxiety in patients with cystic fibrosis and parent caregivers: Results of the International Depression Epidemiological Study across nine countries. Thorax. 2014;69(12):1090-7. doi: http://dx.doi.org/10.1136/thoraxjnl-2014-205983

24. Grossoehme DH, Szczesniak RD, Britton LL, Siracusa CM, Quittner AL, Chini BA, et al. Adherence determinants in cystic fibrosis: Cluster analysis of parental psychosocial, religious, and/or spiritual factors. Ann Am Thorac Soc. 2015;12(6):838-46. doi: http://dx.doi.org/10.1513/Annal sATS.201408-379OC 